ANALYSIS OF INTERPLANETARY DUST COLLECTIONS

\author{
D.E. Brownlee, L. Pilachowski, E. 01szewski, and P.W. Hodge \\ Department of Astronomy \\ University of Washington 98195
}

Abstract - Interplanetary dust particles collected in the form of micrometeorites in the stratosphere and meteor ablation spherules in deep sea sediments are possibly a relatively unbiased sample of the micrometeoroid complex near 1 AU. Detailed laboratory analysis of the particles has provided information on physical properties which may be useful in modeling a variety of aspects of interplanetary dust.

\title{
INTRODUCTION
}

The international effort to analyze lunar samples and meteorites has resulted in development of a wide variety of sophisticated techniques for laboratory study of early solar system materials. During tine past decade, these techniques were used with extraordinary success in investigation of the physical nature and evol ionary histories of the Moon and the meteorite parent bodies. any of these analytical techniques are currently being applied to interplanetary dust samples. At the present iime, laboratory studies are done on micrometeoroids that have successfully entered the Earth's atmosphere. In the near future, it will also $b \in$ possible to analyze the meteorite residue in a large number of microcraters recovered from spacecraft. Ultimately, it is hoped that dust samples will be obtained directly from the sources of the zodiacal cloud: comets and asteroids.

\section{SOURCES OF DUST}

Interplanetary dust is currently available for laboratory investigations from two sources: micrometeorites collected in the stratosphere (Brownlee, 1978) and meteor ablation spherules recovered from deep sea sediments (Blanchard et al., 1979, Brownlee, 1979). Most of the extraterrestrial particles that have been collected in the stratosphere are particles that survived atmospheric entry without melting and are stricto sensu Whipple-style micrometeorites. The stratospheric particles are unfortunately usually limited in size to the range $2 \mu \mathrm{m}-60 \mu \mathrm{m}$. Particles smaller than a micron are essentially uncollectable in the stratosphere because of the enormous background of small terrestrial 
sulfate particles. Cosmic particles $>100 \mu \mathrm{m}$ are exceedingly rare $\left(<10^{-7} \mathrm{~m}^{-3}\right)$ due to both the low extraterrestrial influx and relatively large fall velocities. Such large particles are also strongly heated during atmospheric entry and only particles with high melting points $\left(\sim 2000^{\circ} \mathrm{C}\right)$ can normally survive without melting. Most of the $10 \mu \mathrm{m}$ micrometeorites contain large concentrations of He (Rajan et al., 1977) which presumably is implanted solar wind. Since the implantation depth of solar wind ions is less than a micron, this result suggests that the majority of stratospheric micrometeorites are not fragments of larger bodies but simply interplanetary dust particles that non-destructively entered the atmosphere. The micrometeorite flux obtained by NASA U-2 sampling flights (Figure 1) agrees well with the near Earth meteoroid flux, implying that most of the meteoroid complex near sizes of $10 \mu \mathrm{m}$ probably enters the atmosphere without destruction.

Pristine micrometeorites normally can be collected only in the stratosphere and typical masses of individual particles are on the order $10^{-9} \mathrm{~g}$. Unfortunately, many analytical techniques require particle masses of $10^{-6}-10^{-3} \mathrm{~g}$. Meteoroids of such size normally melt during entry and form meteor ablation spherules. Spheres of $10^{-5} \mathrm{~g}$ mass are very rare and cannot readily be captured before hitting the Earth's surface but they can easily be collected in certain deep sea sediments (Murray and Renard, 1883). In fairly common Pacific sediments, each vertical metre of sediment represents nearly a million years of collection time and for particles $>200 \mu \mathrm{m}$ the sediments often contain only minor amounts of terrestrial particles with magnetic and optical properties similar to cosmic spherules. Most of the cosmic spherules contain ferromagnetic material and are easily extracted from sediments by simple magnetic collectors. The spherules are magnetic because magnetite grains are produced by atmospheric melting and ablation of iron bearing silicates, sulfides or metal (Blanchard et al., 1979). Ferromagnetic spherules are produced from parent materials which originally may not have been magnetic. Deep sea cosmic spherules are not pristine bodies but are altered by neating in the atmosphere and by weathering in the sediment. They do, however, provide individual milligram samples of unique value for determination of certain elemental and isotopic ratios of meteoroids over a size range that includes optical and radar meteors. At the present time there is no information indicating what fraction of the $500 \mu \mathrm{m}$ spherules is produced by meteoroids of similar size and what fraction is produced by destruction of much larger fireball-sized meteoroids.

To date, over 400 stratospheric micrometeorites have been characterized in some detail and over 1000 deep sea spherules examined. It is not known whether or not the examined set of particles is truly representative of near Earth interplanetary dust. It is expected that the particles are fairly representative and it is nearly certain that the most common types of dust should at least exist in the collections. Particle collection does not invclve significant selection effects al though bias to the collections could come from fragmentation and vaporization during entry into the atmosphere. Because of the velocity 


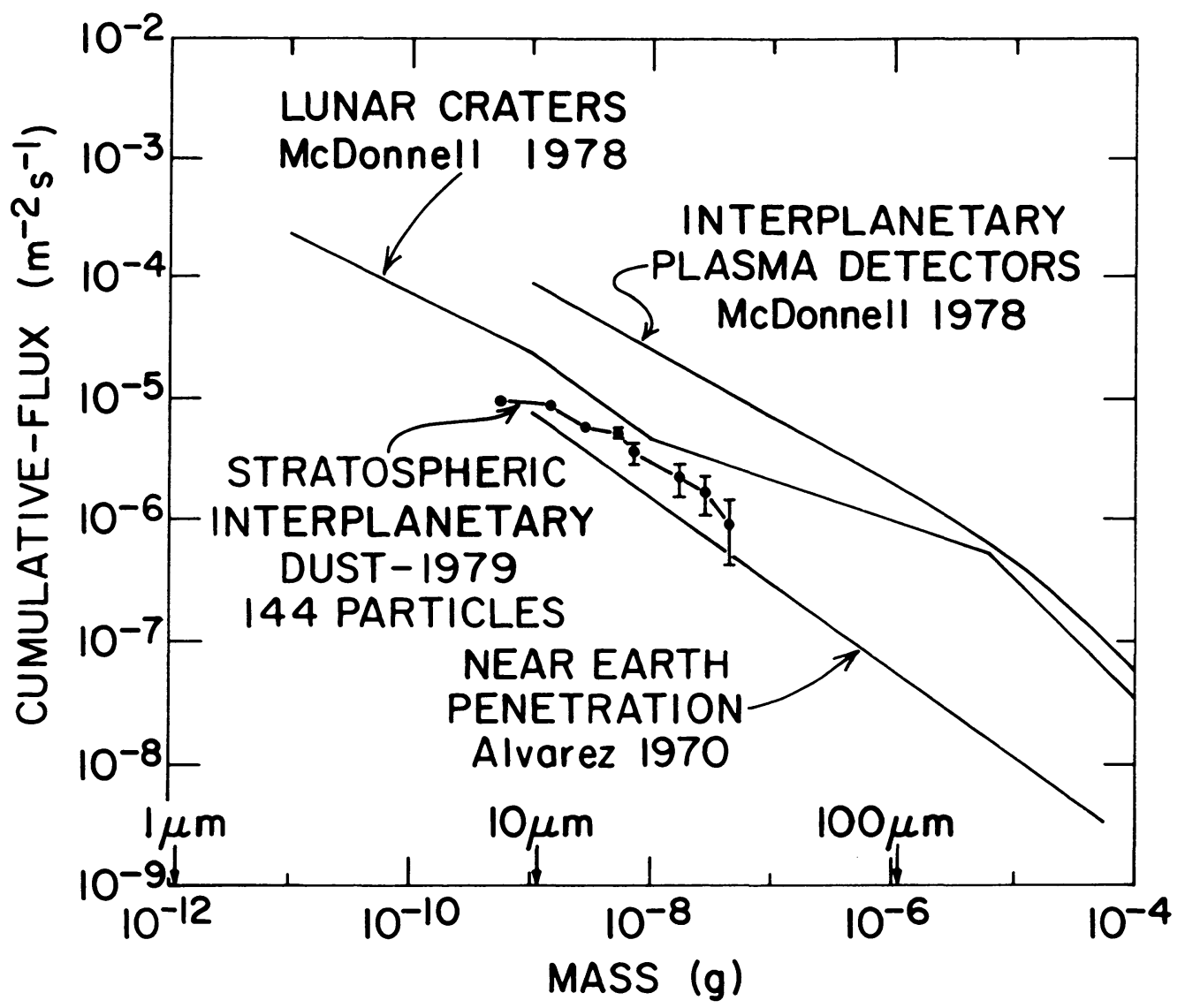

Figure 1. The flux of stratospheric micro meteorites collected by U2 aircraft compared with spacecraft and lunar data.

dependence of atmospheric heating, there certainly should be systematic destruction of high velocity $\left(>30 \mathrm{~km} \mathrm{~s}^{-1}\right)$ meteoroids. Fortunately, typical cosmic dust particles larger than $1 \mu \mathrm{m}$ do not impact the Earth with excessively high velocity. Fragmentation of these small particles should be much less significant than for conventional meteorites. Dust particles decelerate from cosmic velocity at very high altitudes, where the maximum dynamic pressure on the meteoroids is several orders of magnitude less than experienced by meteorite-producing fireballs.

\section{PARTICLE PROPERTIES}

The data from both particle collections indicate that the elemental composition of the majority of dust particles in the size range $5 \mu \mathrm{m}-$ $1000 \mu \mathrm{m}$ is basically chondritic (solar). The data in Figure 2 shows the spread of abundance values for 57 random chondritic particles from the stratosphere. Figure 3 shows abundances determined in unweathered interiors of 60 stony deep sea spherules. Trace element determinations 
by neutron activation also indicate chondritic abundances in both types of particles (Ganapathy et al. 1979a, b). The abundances of the relatively volatile elements $C, S$, and $\mathrm{Zn}$ in the stratospheric particles are higher than those in ordinary chondrites, but are similar to those in CI (type 1) and CM (type 2) carbonaceous chondrites. Within a factor of about two, the stratospheric particles match CI meteorites. There appear to be small systematic deviations from a true CI pattern but this must be considered tentative until a much larger set of particles has been quantitatively analyzed. The chondritic elemental composition of interplanetary dust is not unexpected and was first demonstrated by trace element characterization of the micrometeoroid component of lunar soil (Anders et al., 1973).

The structure of interplanetary particles can be inferred only indirectly from deep sea particles but can be examined directly in the more pristine stratospheric particles. A minor fraction of the deep sea particles do not melt during entry but survive as thermally altered material. The parents of most of these particles appear to have been composed of a fine grained matrix of micron and submicron silicates riddled with small $\mathrm{Ni}$ bearing FeS grains. These particles often contain a few large $(>10 \mu \mathrm{m})$ unmelted grains of enstatite $\left(\mathrm{MgSiO}_{3}\right)$ and forsterite $\left(\mathrm{MgSiO}_{4}\right)$ as minor mineral phases.

The stratospheric particles reveal directly the structures of $10 \mu \mathrm{m}$ sized interplanetary particles. Some $(<40 \%)$ of the particles are solid single mineral grains usually of $\mathrm{FeS}$, forsterite or enstatite. Normally, these single mineral particles are encrusted with fine grained opaque material with chondritic elemental composition. It is clear that in most cases, these particles were originally imbedded in a fine grained matrix of solar-composition material. In addition to the previously mentioned minerals, single mineral grains of FeNi metal, calcite, and Mg phosphate have been collected.

The majority of all collected micrometeorites are black particles with chondritic abundances. These particles are called "chondritic" and occur as at least two types. The rarer type has a smooth exterior, is often depleted somewhat in $\mathrm{Ca}$ and sometimes contains hydrated silicates identifiable by $X$-ray diffraction. These particles are very similar to CI meteorites. The most common are called "chondritic" aggregates and are collections of enormous numbers of grains ranging in size from $<100 \AA$ to several microns (Figure 4). These aggregates are permeated with low atomic number amorphous material (Fraundorf and Shirck, 1979) and appear to be different from known meteoritic materials. In the SEM, most of the mass of the particles appears to be in grains in the $1000 \mathrm{~A}$ to $5000 \mathrm{~A}$ size. Some of these grains however are themselves aggregates of smaller grains. Most of the component grains are fairly equidimensional and appear rounded in the SEM. Fraundorf et al. showed that some of this rounding is due to amorphous coatings. Most of the submicron grains have approximately chondritic abundances for major elements and cannot be single mineral grains. Grains of iron nickel 


\section{U2 CHONDRITIC UNSELECTED AUG,79}

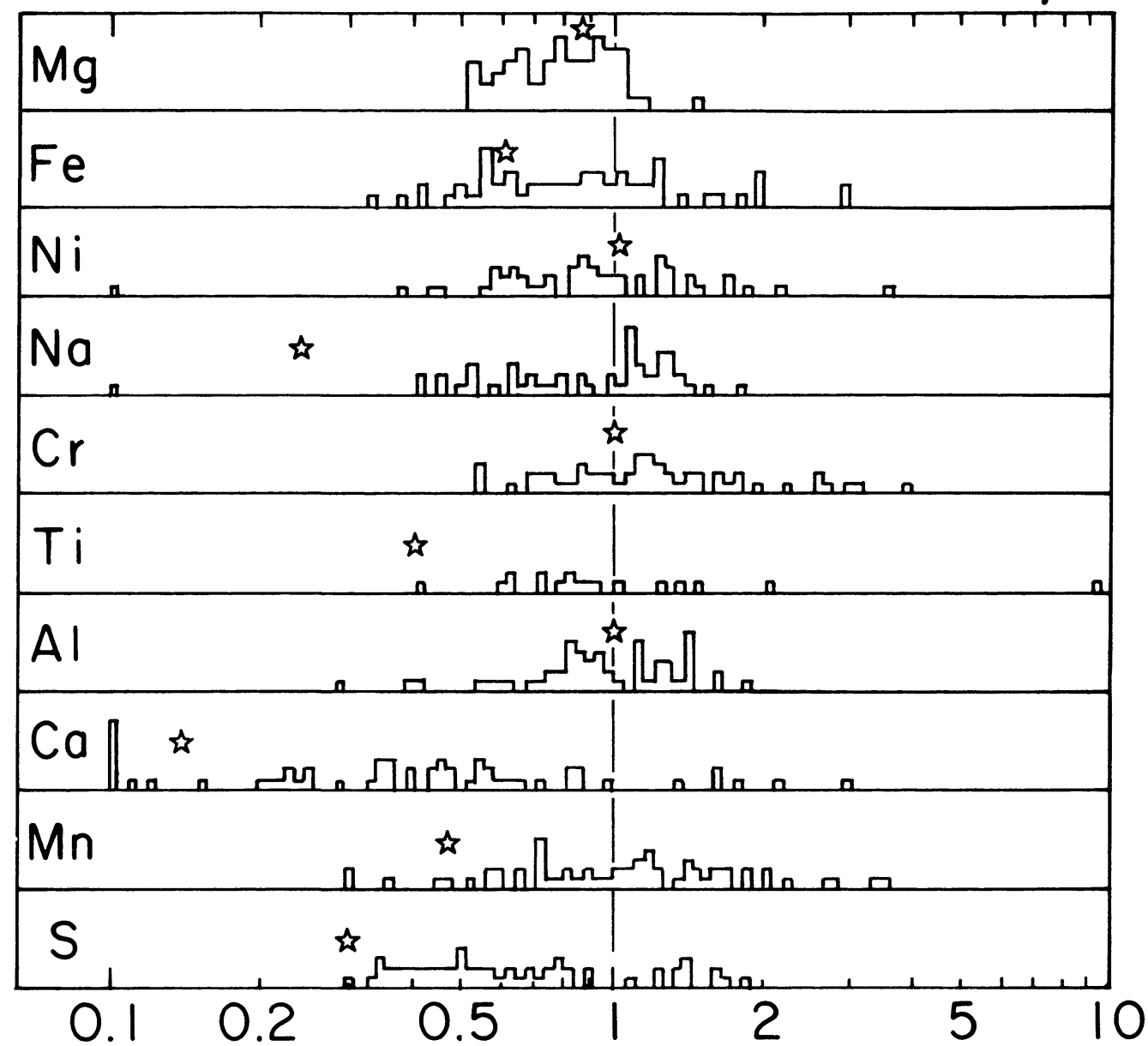

Figure 2. Element to silicon ratios for 57 stratosphere micrometeorites normalized to CI chondrite average. The stars are mean compositions for fine grained matrix of CI meteorites. (McSween and Richardson, 1977).

sulfides are, however, fairly common and grains of olivine and pyroxene do occur. Elemental analysis of 65 grains from a fragmented particle are shown in Figure 5 . It is clear that compositions of the grains do not clump near single mineral compositions.

An intriguing aspect of the chondritic aggregate particles is the observed range in particle density. Some of the aggregates are highly porous with estimated density of unity or possibly less and others are fairly compact with probable density of 2-3. There is a continuous range in particle porosity even though the basic content of the 


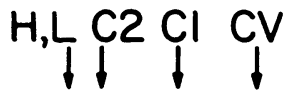

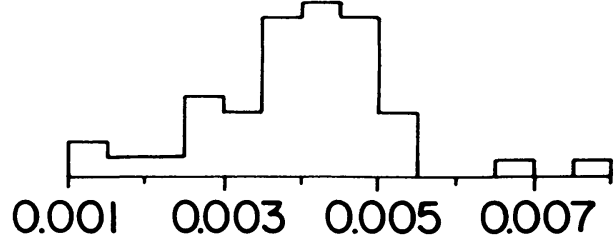

$\mathrm{Ti} / \mathrm{Si}$ (weight percent)

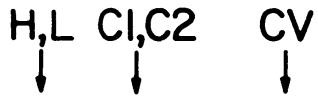

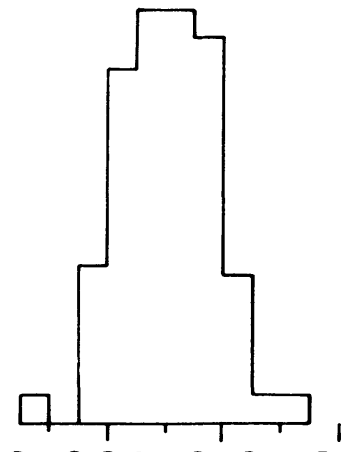

CV C2 $\mathrm{H}, \mathrm{L} \mathrm{Cl}$

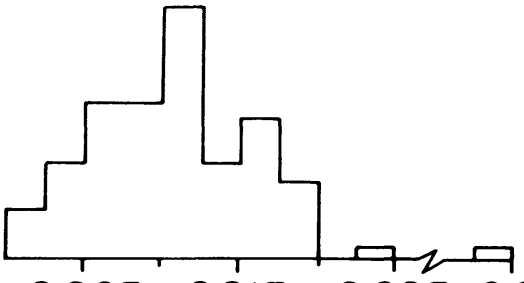

$0.005 \quad 0.015 \quad 0.0250 .040$

$\mathrm{Mn} / \mathrm{Si}$ (weight percent)

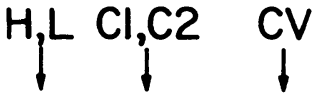

\section{$\begin{array}{llllllll}0.02 & 0.06 & 0.10 & 0.14 & 0.02 & 0.06 & 0.10 & 0.14\end{array}$ $\mathrm{Al} / \mathrm{Si}$ (weight percent) $\mathrm{Ca} / \mathrm{Si}$ (weight percent)}

Figure 3. Elemental abundance ratios measured for unetched interiors of 64 random stony cosmic spherules recovered from deep sea sediments. The histograms are compared with various chondritic meteorite types.

aggregates appears to be the same. This effect could be due to al teration during entry but more probably is a property of the parent bodies. It is highly significant that at least some highly fragile, low density chondritic particles are found. Such materials have not been found in conventional meteorites.

\section{CONCLUSIONS}

Although laboratory studies of micrometeorites and meteor ablation spherules are still in their infancy, they have revealed new insight into the nature of interplanetary dust. As could have been anticipated, the particles are more complex than previously imagined. 


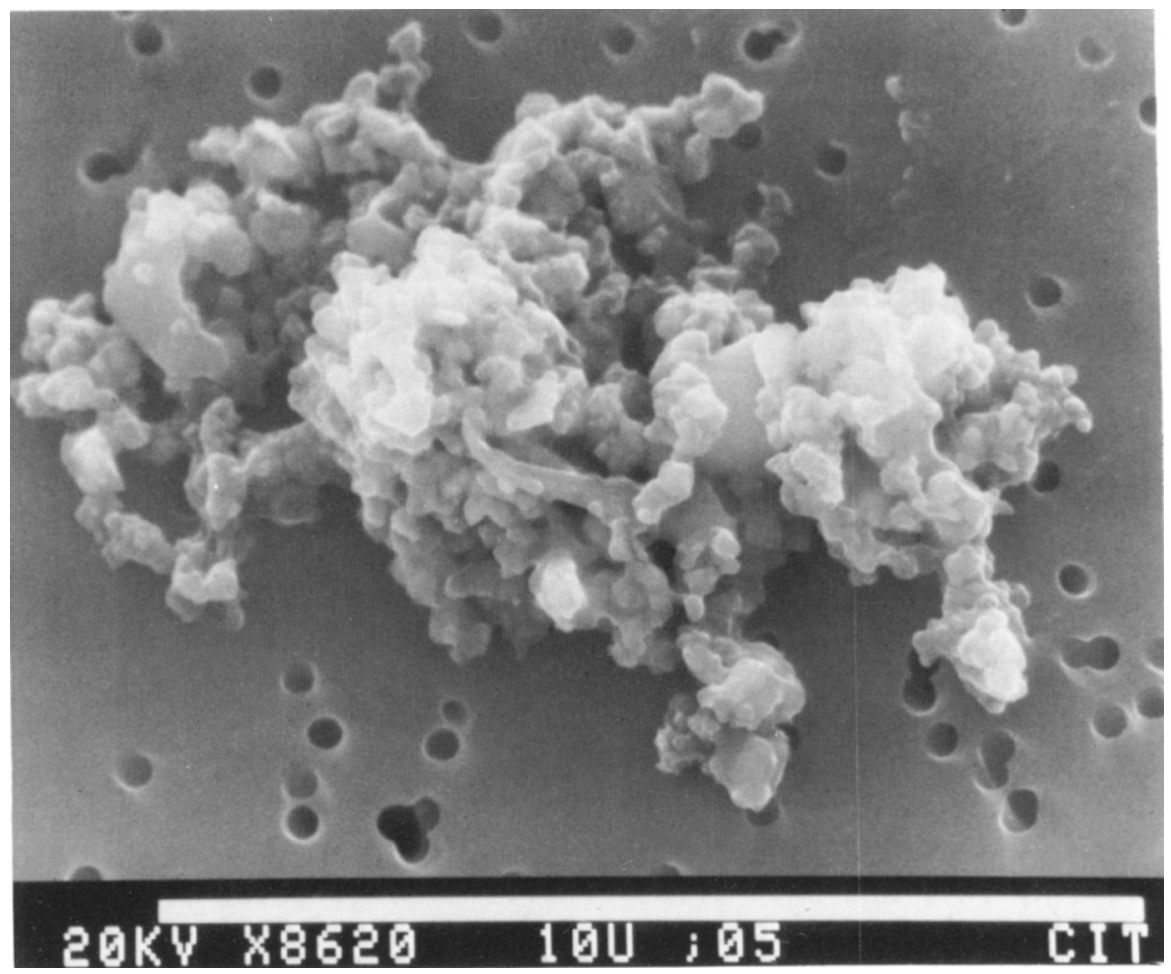

Figure 4. SEM photo of a chondritic aggregate micrometeorite collected in the stratosphere. Scale bar $=10 \mu \mathrm{m}$.

The studies indicate that the majority of $5 \mu \mathrm{m}-50 \mu \mathrm{m}$ particles were produced by the gentle fragmentation of at least two types of parent materials. Both parent materials have volatiTe-rich chondritic compositions, are fine grained and optically black. One parent material is composed of layer-lattice hydrated silicates similar to those in CI and $\mathrm{CM}$ meteorites. The most common particles are products of a different material which has no meteoritic analog. This material is unique because of its porous aggregate structure of relatively equidimensional grains. The particles are complex aggregates of crystalline and amorphous materials and are chemically heterogeneous on a submicron scale. Although most of the grains in the particles are submicron, a small but significant fraction are single mineral grains several microns in size.

Particle production from the interplanetary dust source materials produces a complex of meteoroids with diverse properties. Most of the particles are black clumps of fine grained material. The porosity of these particles varies from near zero to over $50 \%$. The shapes of the particles are somewhat equidimensional al though they are very rough on a scale of one micron because this size is only a few times larger than the common constituents of the aggregates. Particles only a few 


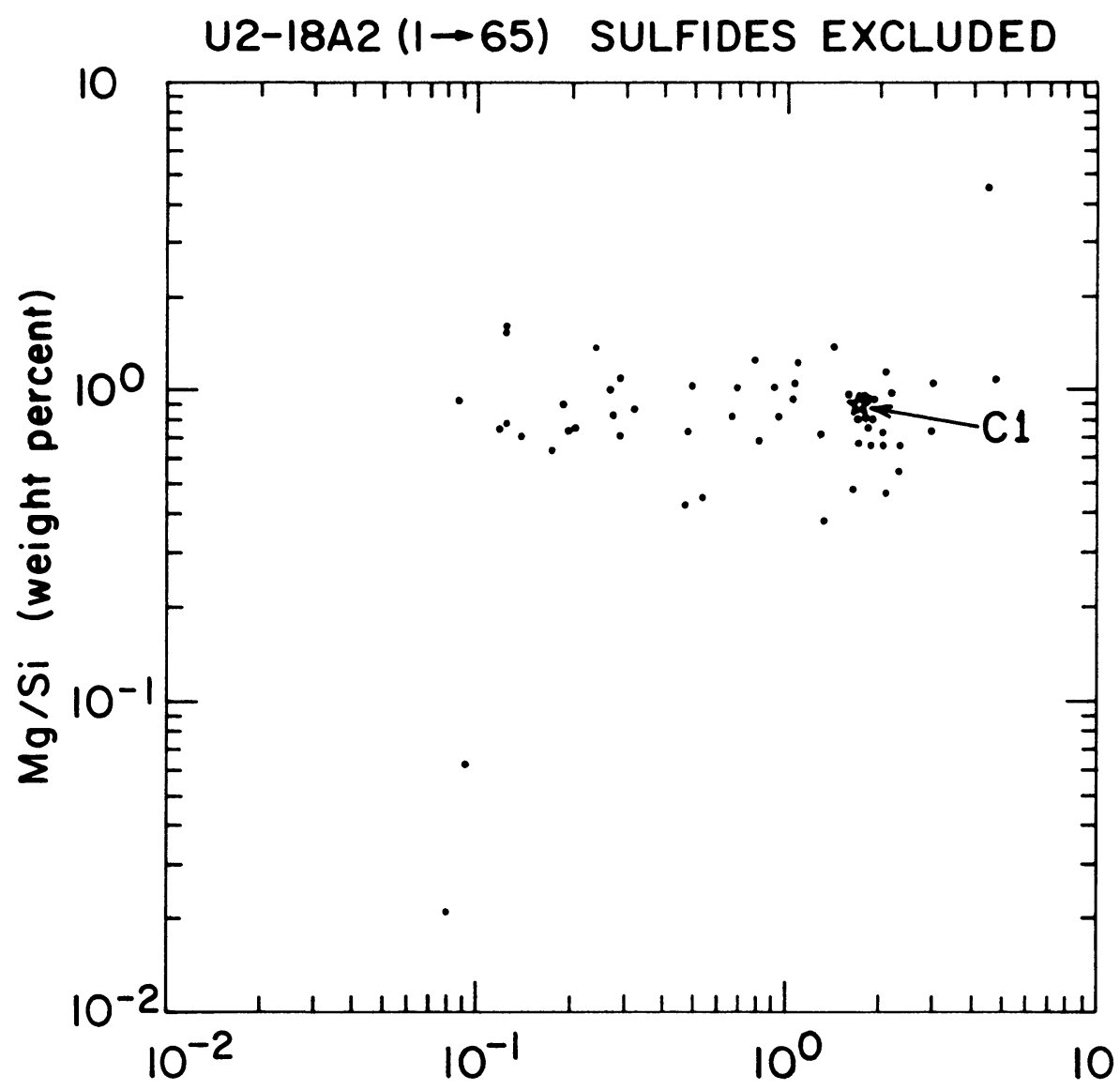

$\mathrm{Fe} / \mathrm{Si}$ (weight percent)

Figure 5. Composition measurements for individual $0.5-2.0 \mu \mathrm{m}$ grains in a fragmented chondritic aggregate micrometeorite. The measurements indicate that these grains are not single minerals. Of special note are the two grains with very high $\mathrm{Si}$; these are probably either $\mathrm{SiC}$ or $\mathrm{SiO}_{2}$.

microns in size have very complicated shapes because they are collections of a relatively small number of individual grains.

In addition to particles made of relatively low density chondritic composition material, the dust parent bodies also produce particles that are relatively dense and essentially are single mineral grains. Normally, these grains are either FeS, olivine or pyroxene. Although the silicate grains are transparent, they contain variable amounts of black, fine-grained chondritic material on their surfaces. The relative 
abundance of chondritic, sulfide, and single mineral silicate particles varies with particle size and is not yet well determined, although it is certain that over $50 \%$ of the particles are chondritic. Metal, magnetite, graphite, or bird-nest aggregates of elongated grains are exceedingly rare.

The collected samples of interplanetary dust, like meteorites, are a "grab sample" of the meteoroid complex. Because atmospheric entry is simple for small particles and because of rapid orbital evolution, it is probable that the dust particles are more representative of the meteoroid complex than are meteorites. This presumption is strengthened by the observation that most of the dust particles are primitive carbonaeous chondrite-like materials while such fragile materials are very rare among meteorite falls.

Acknowledgements - We would like to thank Chris Suver for his help with the computing involved in this work. Much of this work was supported by NASA grant NSG-9052.

\section{REFERENCES}

Anders, E., Ganapathy, R., Krahenbuhl, U.R.S., and Morgan, J.W.: 1973, Moon, 8, pp. 3-24.

Blanchard, M.B., Brownlee, D.E., Bunch, T.E., Hodge, P.W., and Kyte, F.T.: 1979, Earth Planet. Sci. Lett., in press.

Brownlee, D.E.: 1979, in C. Emiliani (ed.), "The SEA", 7, in press.

Brownlee, D.E.: 1978, in J.A.M. McDonnel1 (ed.), "Cosmic Dust", Wiley and Sons, New York, pp. 295-336.

Fraundorf, P. and Shirck, J.: 1979, Proc. Lunar and Planet. Sci. Conf. 10, in press.

Ganapathy, R., Brownlee, D.E., and Hodge, P.W.: 1978, Science, 201 , pp. $1119-1121$.

Ganapathy, R., and Brownlee, E.D.: 1980, Science, in press.

Kessler, D.J.: 1979, NASA SP 8038.

McDonnel1, J.A.M.: 1978, Cosmic Dust, Wiley and Sons, New York.

McSween, H.Y., and Richardson, S.M.: 1977, Geochim. Cosmochim. Acta, 41, pp. $1145-1161$.

Murray, J., and Renard, A.F.: 1883, Proc. Roy. Soc. Edinburg, 12 , pp. 474-495.

Rajan, R.S., Brownlee, D.E., Tomandl, D., Hodge, P.W., Farrar, H., and DISCUSSION Britten, R.A.: 1977, Nature 267, pp. 133-134.

Hughes: Could you explain in more detail how you obtained the spherules? Brownlee: The particles were collected by towing a $300 \mathrm{~kg}$ magnet across the sea floor at a depth of $5000 \mathrm{~m}$. Remote ocean sites distant from volcanoes and sediment fans from continents do not usually contain terrestrial particles that are magnetic and larger than about $200 \mu \mathrm{m}$. Extraterrestrial material is concentrated in sediments because the accumulation rate of terrestrial material is only about $1 \mu \mathrm{m}$ per year.

Lamy: 1) Regarding the amorphous coating you mentioned on the individual grains in aggregates, does it resemble that on lunar grains due to solar wind implantation? 2) In a previous publication you favoured a composition 
close to ordinary chondrites; your new analys is appears now to favour a composition close to $\mathrm{C} 1$ and $\mathrm{C} 2$.

Brownlee: 1) The amorphous coatings in stratospheric particles are low atomic number material, while on lunar grains the composition of the coating is the same as the host. 2) The abundances of the volatile elements of the dust particles are similar to $\mathrm{Cl}$ and $\mathrm{C} 2$ meteorites.

Singer: To what extent does your experimental technique introduce a selection bias? For example, the ambient concentration at the collection level is inversely related to rate of fall and therefore density times radius. What about the collection process itself? Are you missing a significant fraction of the incoming particles?

Brownlee: In the size range $5 \mu \mathrm{m}-25 \mu \mathrm{m}$, there are no obvious selection effects. Particles smaller than $10 \mu \mathrm{m}$ with exceedingly low density, $<10^{-2} \mathrm{~g} \mathrm{~cm}^{-3}$, would probably blow around the collector and not be captured.

MiZZman: In your Heidelberg paper (1975) you mentioned that the chondritic aggregate type of particle accounted for approximately $60 \%$ of your cosmic dust particle collection. Does this figure still hold? Brownlee: It varies with size but at $10 \mu \mathrm{m}$ about $60 \%$ of the particles are chondritic in composition.

Giese: For investigating optical scattering properties by microwave simulation experiments, I would like to get a description of the overall optical appearance of a "typical Brownlee particle". What percent of the total volume is a) filled by black components, b) filled by transparent components, and c) empty?

Brownlee: This varies from particle to particle and of course is difficult to measure quantitatively. For the porous particles the ratios are about; $50 \%$ void, $45 \%$ black, and 5\% transparent grains of micron size.

Alexander: Do you feel that any submicron particles are seen that are not from breakups? Can you see any kind of size cutoff? Or can you not distinguish between . $5 \mu \mathrm{m}$ from interplanetary space and $.5 \mu \mathrm{m}$ from breakup? Brownlee: There is no size cutoff. Particles resulting from breakup near the Earth of larger bodies should not contain implanted solar wind.

Fechtig: Did you find any lunar dust in your collections?

Brown lee: It may be possible to identify lunar spherules in deep sea sediments. This is more difficult than identifying chondritic particles because of the range of composition of lunar materials and their similarity to some terrestrial particles. The undifferentiated chondritic material is quite different from common terrestrial particles.

Grün: What is the density and percentage of the fluffy particles? Brownlee: The density can only be estimated, but based on porosity the density range for chondritic particles is from about 3 down to somewhat less than $1 \mathrm{~g} \mathrm{~cm}^{-3}$. About $25 \%$ of the chondritic particles are porous. 